\title{
LUMINESCENCE FROM SMALL CARBONACEOUS DUST GRAINS
}

\author{
Christian Friedemann and Hans-Georg Reimann \\ Jena University Observatory \\ Schillergaesschen 2 \\ 6900 Jena \\ GDR
}

\begin{abstract}
A relationship exists between the amount of the ultraviolet (UV) extinction and the relative strength of the very broad structure (VBS) in the $500-600 \mathrm{~nm}$ wavelength region $(5000-6000 \AA)$ of the interstellar extinction curve. It is based on observations of $O B$ stars distributed along the Milky Way. By examining $\mathrm{E}(\mathrm{B}-\mathrm{V})$, we found that larger-than-mean values of the UV extinction correspond to larger-than-mean strengths of the VBS and vice versa. This feature is thought to be an emission arising from photoluminescence (PL) of small amorphous carbonaceous grains.
\end{abstract}

\section{INTRODUCTION}

Whiteoak (1966) first investigated the shallow dip (the VBS) in the 500-600 nm (5000-6000 $\AA$ ) spectral region in the interstellar extinction curve. Recently, Krelowski, Maszkowki, and Strobel (1986) studied the profile of the VBS spectrophotometrically and found three substructures centered at about 540, 590, and $680 \mathrm{~nm}$. Reimann and Friedemann (1989) accomplished a filterphotometric method for measuring the strength of the VBS originally proposed by Reimann (1986). The relative strength of the VBS according to

$$
\Delta \mathrm{E}=[\mathrm{E}(b-y) / \mathrm{E}(B-V)]_{\mathrm{obs}}-\mathrm{E}(b-y) / \mathrm{E}(B-V)_{\text {spectraltype }}
$$

is the difference between the observed color excess ratios and the spectral type-dependent means.

\section{CORRELATION OF THE VBS WITH THE CONTINUOUS UV EXTINCTION}

Van Breda and Whittet (1981) and Reimann (1986) pointed out that there is only a loose connection between the strength of the VBS and that portion of grains (larger) that cause the visual extinction. The question arises whether there exists any correlation between the strength of the VBS and that share of particles (smaller) responsible for the far-ultraviolet (FUV) extinction. Its amount can be described by a parameter introduced by Gürtler et al. (1982) in a simple analytical approximation of the shape of the curve of the UV extinction. The UV parameter $\alpha_{\text {rel }}$ is defined by

$$
\alpha_{\text {rel }}=\left(\alpha-\alpha_{\text {cal }}\right) / E(B-V) .
$$

In this equation, $\alpha_{\text {cal }}$ denotes calculated mean values according to an empirical relation $\alpha \approx \mathrm{E}(B-V)$.

In our study, such lines of sight in which the continuous UV extinction deviates from the average one are of interest. The correlation (see Figure 1) means that a lower-than-average UV extinction is connected with a weaker-than-normal strength of the VBS and vice versa. 
Figure 1. Relation between the amount of UV extinction $\alpha_{\text {Tel }}$ and the relative strength $\Delta \mathrm{E}$ of the VBS. Various symbols indicate memberships of the stars to specific associations. (Note: Open circles mark several associations for which only one or two stars each were found.)

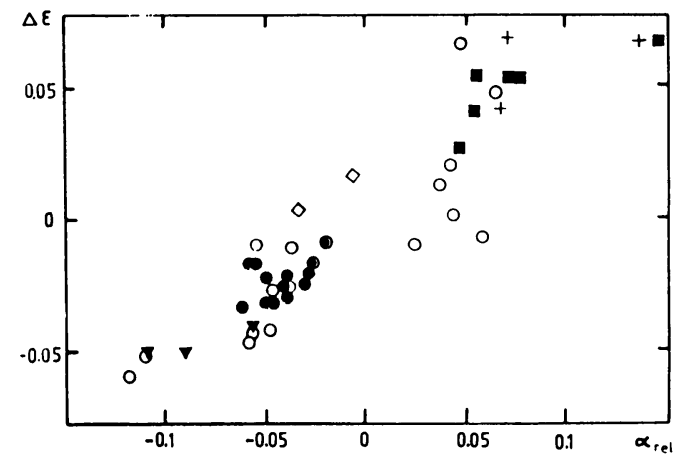

\section{ATTEMPTS TOWARD A PHYSICAL EXPLANATION OF THE CORRELATION}

Up to now, a commonly accepted explanation of the origin of the VBS has not existed. Reimann (1986) proposed that the VBS is probably an emission feature. The VBS could possibly arise from a kind of luminescence in disordered structures of the interstellar grains-an explanation also proposed for the extended red emission mostly observed in reflection nebulae. PL is a possible physical process that could explain the VBS emission. Laboratory work (e.g., Guzzi et al. 1987; Watanabe, Hasegawa, and Kurata 1982; Wdowiak et al. 1989) has been performed for amorphous silica and carbonaceous substances. These probes show that PL in HAC originates from direct radiative transitions from the conduction band tail to the valence band tail corresponding to the general model proposed by Street (1981). Therefore, the peak wavelength of the luminescence band measured in HAC should be related more to the electronic structure than to impurities or defects in the carbonaceous material. HAC could be realized as a kind of "alloy" consisting of linear, planar, and three-dimensional crystalline structures such as acethylene, graphitic, and diamondlike bonded C atoms. Duley (1984) showed that an equal-sized diamondlike structure has a higher absorption efficiency than a graphitic one. This observation gives rise to the assumption that the ratio of $\mathrm{sp}^{2}$-to-sp ${ }^{3}$ bonding structures in HAC determines the FUV extinction as well as the wavelength of the PL peak. The correlation found (Figure 1) could indicate that an increasing strength of the VBS is connected with a larger fraction of three-dimensional structures in the HAC dust.

\section{REFERENCES}

Duley, W. W. 1984, Ap. J., 287, 694.

Gürtler, J. et al. 1982, Astron. Nachr., 303, 105.

Guzzi, M. et al. 1987, Phys. Rev. b, 35, 9407.

Krelowski, J., Maszkowki, R., and Strobel, A. 1986, Astro. Ap., 166, 271.

Reimann, H.-G. 1986, in The Role of Dust in Dense Regions of Interstellar Matter, eds. T. Henning et al., p. 83.

Reimann, H.-G. and Friedemann, C. 1989, Astro. Ap., submitted.

Street, R. A. 1981, Adv. in Phys., 30, 593.

Van Breda, I. G. and Whittet, D. C. B. 1981, MNRAS, 195, 79.

Watanabe, I., Hasegawa, S., and Kurata, Y. 1982, Japanese J. Appl. Phys., 21, 856.

Wdowiak, Th. et al. 1989, Ap. J., 336, 838.

Whiteoak, J. B. 1966, Ap. J., 144, 305. 